\title{
Effect of Attapulgite-Doped Electrospun Fibrous PLGA Scaffold on Pro-Osteogenesis and Barrier Function in the Application of Guided Bone Regeneration
}

This article was published in the following Dove Press journal:

International Journal of Nanomedicine

\begin{abstract}
Xinru Xie, ',* Xiangyang Shi, (iD ${ }^{2, *}$ Shaoyi Wang,' Lingyan Cao, ${ }^{3}$ Chi Yang (D)' Zhigui Ma,'

'Department of Oral Surgery, Shanghai Ninth People's Hospital, College of Stomatology, Shanghai Jiaotong University School of Medicine; National Clinical Research Center for Oral Diseases; Shanghai Key Laboratory of Stomatology \& Shanghai Research Institute of Stomatology, Shanghai, People's Republic of China; ${ }^{2}$ College of Chemistry, Chemical Engineering and Biotechnology, Donghua University, Shanghai, People's Republic of China; ${ }^{3}$ Department of Prosthodontics, Shanghai Ninth People's Hospital, College of Stomatology, Shanghai Jiaotong University School of Medicine; National Clinical Research Center for Oral Diseases; Shanghai Key Laboratory of Stomatology \& Shanghai Research Institute of Stomatology, Shanghai, People's Republic of China

*These authors contributed equally to this work
\end{abstract}

Correspondence: Chi Yang; Zhigui Ma Department of Oral Surgery, Shanghai Ninth People's Hospital, College of Stomatology, Shanghai Jiaotong University School of Medicine; National Clinical Research Center for Oral Diseases; Shanghai Key Laboratory of Stomatology \& Shanghai Research Institute of Stomatology, Shanghai, People's Republic of China Emailyangchi63@hotmail.com; mazhigui2007@I26.com
Purpose: Guided bone regeneration (GBR) therapy, which is a widely used technique in clinical practice and is effective in improving the repair of alveolar bone defects or bone mass deficiency regeneration, requires the use of membrane materials with good biocompatibility, barrier function, rigidity matching the space maintenance ability, economic benefits and excellent clinical applicability. The aim of this study was to develop an electrospun attapulgite (ATT)-doped poly (lactic-co-glycolic acid) (PLGA) scaffold (PLGA/ATT scaffold) as a novel material for GBR applications.

Methods and Results: Scanning electron microscopy (SEM) and X-ray diffraction (XRD) were used to determine the morphology and the crystalline structure of the PLGA/ATT scaffolds, respectively. Porosity and contact-angle measurements were also carried out to further characterize the physical properties of the PLGA/ATT scaffolds. The results of in vitro studies showed that bone marrow mesenchymal stem cells (BMSCs) attached more readily to and spread better over the PLGA/ATT scaffolds than the Bio-Gide membrane. Furthermore, in the in vitro osteoinductive experiments with BMSCs, the PLGA/ATT scaffolds were found to enhance the activity of alkaline phosphatase (ALP), promote the formation of mineralized bone nodules, and up-regulate the expression of several osteogenic markers - namely, runt-related transcription factor 2, alkaline phosphatase, osteopontin, and osteocalcin - which are similar to the effects of the Bio-Gide membrane. Further, in in vivo studies, the results of sequential fluorescent labeling, micro-computed tomography, and histological analysis suggest that using the PLGA/ATT scaffolds for repairing V-shaped buccal dehiscence on a dog's tooth root improved bone regeneration, which is not only similar to the result obtained using the Bio-Gide membrane but also much better than that obtained using PLGA scaffolds and the negative control.

Conclusion: To achieve satisfactory therapeutic results and to lower the cost of GBR treatment, this study provided a promising alternative material of bio-degradable membrane in clinical treatment.

Keywords: electrospun, guided bone regeneration, attapulgite, osteogenesis

\section{Introduction}

Fenestration and dehiscence are localized deficiencies of the facial or lingual cortical plate that covers the teeth. ${ }^{1-3}$ Studies have showed that alveolar bone defects are common in various malocclusions, possibly causing consequent gingival recession and exacerbating loss during orthodontic treatment. ${ }^{4,5}$ Therefore, it is

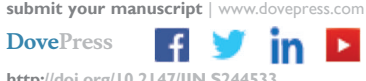

http:/10iorg/10.2147un 3244533
International Journal of Nanomedicine 2020:15 676I-6777 
important to increase the bone volume before the orthodontic treatment. The guided bone regeneration (GBR) treatment, in which great advances have been made in recent years, is regarded to be a successful and reliable technique for vertical and horizontal bone ridge augmentation. ${ }^{6,7}$ To achieve adequate bone augmentation, a mechanical barrier is essential; this barrier will protect against blood clots and isolate the bone defect from the surrounding connective tissue, thereby enhancing the proliferation and differentiation of bone cells, and eventually promoting bone defect repair. ${ }^{8,9}$ An ideal GBR membrane should meet the requirements of biocompatibility, barrier action, appropriate stiffness for space maintenance, and clinical manageability. ${ }^{10}$ However, most membranes cannot fulfill all requirements to be ideal membranes for the GBR technique. Among the commercial products, collagen membranes (such as the Bio-Gide membrane) have been most widely utilized. However, these have a high price and low tensile strength, which limits their application in clinical treatment. ${ }^{11}$

Electrospinning is a simple, flexible, and cost-effective nanofabrication technique that can be used to fabricate ultralong nanofibers with controllable diameters ranging from a few nanometers to several micrometers. ${ }^{12}$ Nanoscale fibers manufactured by this technique can mimic the extra-cellular matrix (ECM) structure with a large surface area and loosely connected 3D porous structure, and this makes the fibers efficient for tissue engineering applications. ${ }^{13}$

Poly(lactic-co-glycolic acid) (PLGA) is an FDAapproved biodegradable polymer and can be electrospun to form nanofibers. It is attracting considerable interest because of its controllable microstructure and degradation rate. ${ }^{14,15}$ However, its disadvantages include poor hydrophilicity, cellular affinity, and local inflammatory response caused by the products of acidic degradation of PLGA. ${ }^{16}$ In addition, electrospun neat-PLGA membranes lack sufficient mechanical strength needed for GBR applications. ${ }^{17}$ Furthermore, the fibrous matrix with a high porosity may not prevent fibroblast infiltration. The efficiency of electrospun fibers will be further affected by bioactive agents incorporated into artificial ECM structures.

Attapulgite (ATT; chemical formula: $(\mathrm{Mg}, \mathrm{Al})_{2} \mathrm{Si}_{4} \mathrm{O}_{10}$ $\left.(\mathrm{OH}) \cdot 4\left(\mathrm{H}_{2} \mathrm{O}\right)\right)$ is a natural clay material, a type of magnesium aluminum phyllosilicate that is widely distributed in China and the United States. ATT/polymer composite materials are expected to have better mechanical durability than the corresponding neat polymers. Tian et $\mathrm{al}^{18}$ reported that a small mass fraction of ATT improved the mechanical properties substantially. In our previous work, we have shown that the doped ATT within the PLGA nanofibers is able to induce the osteoblastic differentiation of human mesenchymal stem cells, even without the stimulating factor resulting from dexamethasone. However, to our knowledge, in vivo studies on the suitability of electrospun ATT-doped PLGA fibrous scaffolds for repairing alveolar bone defects have not been conducted.

We hypothesized that the electrospun ATT-doped PLGA fibrous scaffold could induce the expression of osteogenic factors in bone marrow mesenchymal stem cells (BMSCs) and effectively promote bone formation in alveolar defects. To verify our hypothesis, in this study, electrospun ATT-doped PLGA fibrous scaffolds were prepared and analyzed in vitro in terms of their physicochemical properties, bioactivity, and osteoinduction. Moreover, the electrospun ATT-doped PLGA fibrous scaffolds were applied to repair alveolar dehiscence in dogs, and their effectiveness for bone regeneration was assessed by performing sequential fluorescent labeling, micro-computed tomography (micro-CT) measurements, and the histological analyses after 12 weeks in vivo. To the best of our knowledge, these are the first experimental data reporting healing at dehiscence by using the PLGA/ ATT scaffold in the GBR procedure.

\section{Materials and Methods}

\section{Fabrication of Electrospun ATT-Doped PLGA Fibrous Scaffold}

Electrospun PLGA fibers $\left(\mathrm{Mw}=81,000 \mathrm{~g} \cdot \mathrm{mol}^{-1}\right)$ with a lactic acid/glycolic acid ratio of 50:50 and PLGA-3\% ATT fibers was fabricated according to our previously published procedures. ${ }^{19}$ Briefly, PLGA was dissolved in a solvent mixture of tetrahydrofuran (THF) and dimethylformamide $(\mathrm{DMF})(\mathrm{v} / \mathrm{v}=3: 1)$ to obtain a homogeneous solution with a concentration of $25 \%(\mathrm{w} / \mathrm{v})$. Then, $3 \%$ ATT was dissolved in the PLGA solution and stirred for $1 \mathrm{~h}$ to obtain a homogeneous solution. The electrospinning apparatus consisted of a syringe pump, high-voltage power supply and a thin aluminum foil. The electrospinning solution was fed using an infusion pump into a $10 \mathrm{~mL}$ plastic syringe fitted with a stainless-steel blunt needle with a $0.8-\mathrm{mm}$ diameter and an injection speed of $0.8 \mathrm{~mL} / \mathrm{h}$. A fixed electrical potential of $30 \mathrm{kV}$ was applied to the composite solution. Randomly oriented nanofibers were collected on a flat collector that was wrapped with an 
aluminum foil and kept at a distance of $15 \mathrm{~cm}$ from the needle tip. The scaffolds were dried for at least $48 \mathrm{~h}$ under vacuum at $25^{\circ} \mathrm{C}$ to remove the residual solvent. To make a comparison with the Bio-Gide used in clinical practice, the thickness of the electrospun PLGA and PLGA/ATT that we fabricated was similar, excluding the impact of thickness on osteogenesis.

\section{Characterization of ATT-Doped PLGA Electrospun Scaffold}

Thickness was measured with a digital calliper (Rupec 239, resolution $0.001 \mathrm{~mm}$, reference standard DIN 863/ 1). The morphology of the developed scaffold was detected by performing scanning electron microscopy (SEM; S-3400; Hitachi, Tokyo, Japan) with an accelerating voltage of $15 \mathrm{kV}$ via gold sputter coating. The diameters of the nanofibers were analyzed using software ImageJ. The crystalline structure of the scaffolds was evaluated by performing X-ray diffraction (XRD; X PertPro MPD system, PANalytical B.V, Almelo, the Netherlands) at $25^{\circ} \mathrm{C}$ by using $\mathrm{Cu} \mathrm{Ka}$ radiation in the $2 \theta$ range from $10^{\circ}$ to $70^{\circ}$, and a scanning speed of $2 \% \mathrm{~min}$. To determine the porosity of the fibrous samples, three small strips $\left(20 \times 20 \mathrm{~mm}^{2}\right)$ were cut from the center of nanofibrous mats, and the porosity of the fibrous mats was calculated by using the following equations:

$$
\begin{gathered}
\rho_{a}\left(\mathrm{gcm}^{-3}\right)=\frac{m(\mathrm{~g})}{d(\mathrm{~cm}) \times s\left(\mathrm{~cm}^{2}\right)} \\
p=1-\frac{\rho_{a}\left(\mathrm{gcm}^{-3}\right)}{\rho_{b}\left(\mathrm{gcm}^{-3}\right)} \times 100 \%
\end{gathered}
$$

where $\rho_{a}$ and $\rho_{b}$ stand for the apparent density and bulk density, respectively, of the PLGA or electrospun ATTdoped PLGA fibrous scaffolds; and $\mathrm{p}, \mathrm{m}, \mathrm{d}$, and s stand for the porosity, mass, and area of the strip, respectively. Note that the bulk density of the ATT-doped PLGA nanofibrous scaffold was calculated based on the contents of PLGA $\left(1.25 \mathrm{~g} \mathrm{~cm}^{-3}\right)$ and ATT $\left(2.18 \mathrm{~g} \mathrm{~cm}^{-3}\right)$. The surface hydrophilicity of the PLGA and ATT-doped PLGA fibrous mat was evaluated by measuring the water contact angles. Three $1 \mathrm{~mL}$ pendant droplets of distilled water were dropped onto the surface of each sample at a randomly selected location at the ambient temperature and humidity. The contact angle was measured by using a contact angle goniometer (DSA-30, Kruss, Germany) when the droplets were stable.

\section{Mechanical Properties}

The PLGA, PLGA/ATT scaffolds and the Bio-Gide membrane were cut into rectangular strips with the dimension of $5 \mathrm{~mm} \times 40 \mathrm{~mm}$. The tensile properties were performed with the All-purpose Electronic Tester (ENGYI, China) equipped with a 100-N load cell. To evaluate the stability of the PLGA, PLGA/ATT scaffolds and the Bio-Gide membrane, we placed the samples into $20 \mathrm{~mL}$ PBS $(\mathrm{PH}=7.4)$ and immersed in vitro at $37^{\circ} \mathrm{C}$ for 1 day and 7 days. Then, the immersed and non-immersed samples were mounted into the grips separately with a gauge length of $10 \mathrm{~mm}$ and were stretched under the cross-head speed of $5 \mathrm{~mm} / \mathrm{min}$ until breakage to obtain the stress-strain curves. The Young's modulus of elasticity (E), ultimate tensile strength (UT) and elongation at break could be analyzed by the stress-strain curves.

\section{Ethics Statement}

All experimental protocols regarding the use of animals in this study were approved by the Institutional Animal Care and Use Committee, and we followed the procedure for Animal Experimental Ethical Inspection of the Ninth People's Hospital, which is affiliated with the Shanghai Jiao Tong University School of Medicine.

\section{BMSC Culture}

Primary BMSCs were harvested from 4-week-old Sprague-Dawley rats (Shanghai SLAC Experimental Animal Center, Shanghai, People's Republic of China). The bone marrow was flushed out with DMEM (HyClone, Logan, UT, USA) supplemented with 1\% penicillin/streptomycin (HyClone, Logan, UT, USA). After blood cells were removed by centrifugation, they were mixed with complete DMEM supplemented with 10\% fetal bovine serum (FBS; HyClone, Logan, UT, USA) and plated into a tissue culture flask. When large colonies formed and became confluent, cells were trypsinized with 10\% trypsin-EDTA (HyClone, Logan, USA) and passaged. BMSCs acquired from passages 2 and 3 were used for our experiments.

\section{Morphology, Adhesion, and Growth of Seeded BMSCs}

Rat BMSCs with a density of $1.0 \times 10^{5}$ cells $/ \mathrm{mL}$ were cultured on PLGA, PLGA/ATT, and Bio-Gide scaffolds (each $15 \mathrm{~mm}$ in diameter) in 24-well plates. Three specimens per each group were tested for SEM after 24 hour- 
cell seeding $(n=3)$. The samples were fixed in $2.5 \%$ glutaraldehyde overnight at $4{ }^{\circ} \mathrm{C}$, washed thrice with PBS, and then dehydrated using increasing ethanol concentrations. Finally, these samples were dried using hexamethyldisilazane, sputter-coated with gold, and examined by SEM.

\section{Cell Proliferation Assay}

The viabilities of BMSCs proliferated on the membrane were assessed by using the Cell Counting Kit-8 (CCK8; Dojin, Shanghai, China), according to the manufacturer's protocol. BMSCs were seeded onto the samples (15 $\mathrm{mm}$ in diameter) at a density of $5.0 \times 10^{3}$ cells/well in 96-well plates. Then, after 1, 3, and 5 days of incubation, the membranes were washed thrice with PBS to remove non-attached cells, and then $10 \mu \mathrm{L}$ of CCK-8 solution was added to each well, followed by cell incubation for $2 \mathrm{~h}$ at $37^{\circ} \mathrm{C}$. The absorbance was determined at a wavelength of $450 \mathrm{~nm}$. All experiments were performed in triplicate.

\section{Alkaline Phosphatase (ALP) Activity Assay and Alizarin Red-S (ARS) Staining}

Cells were seeded on the membrane (15 $\mathrm{mm}$ in diameter) with a density of $1.5 \times 10^{5}$ cells $/ \mathrm{mL}$ in 24 -well plates, and ALP staining was performed by using the ALP staining kit (Beyotime, Suzhou, China) at 1 and 7 days. The quantitative ALP activity was determined using $p$-nitrophenyl phosphate (pNPP; Beyotime, Suzhou, China). The absorbance values (OD) were recorded at a wavelength of 405 $\mathrm{nm}$ to determine the ALP activity. The total protein content was assessed by using a BCA protein assay kit (Sigma, USA), and the OD values were normalized to the bovine serum albumin (BSA; Sigma, USA) standard curve at $590 \mathrm{~nm}$. Then, the level of pNPP production was assessed at the OD value of $405 \mathrm{~nm}$. After osteogenic induction in the osteogenic medium for 21 days, the mineral deposition by cultured BMSCs was assessed by Alizarin Red S staining: The cells were washed thrice with PBS, fixed in 70\% ethanol for $30 \mathrm{~min}$, and stained with Alizarin Red S (ARS; Sigma, USA) for $30 \mathrm{~min}$ at $37^{\circ} \mathrm{C}$.

\section{Real-Time Polymerase Chain Reaction (PCR) Assay}

BMSCs were seeded on the scaffold (15 $\mathrm{mm}$ in diameter) in 6-well plates at a density of $1 \times 10^{5}$ cells $/ \mathrm{mL}$, and total RNA isolation after various treatments was realized by using the Trizol reagent (Invitrogen, Carlsbad, NM, USA). Complementary DNA (cDNA) was synthesized by using a cDNA Synthesis Reverse Transcription Kit (Fermentas, Thermo, USA). The real-time PCR assays for runt-related transcription factor 2 (Runx2), alkaline phosphatase (ALP), osteopontin (OPN), and osteocalcin $(\mathrm{OCN})$ were performed by using a Light-Cycler system with SYBR Premix Ex Taq ${ }^{\mathrm{TM}}$ (Takara, Japan) according to the manufacturer's instructions. The relative gene expressions were analyzed by using the comparative $\mathrm{Ct}\left(2^{-\Delta \Delta \mathrm{Ct}}\right)$ method. The data are expressed as the fold change relative to untreated controls after normalization to the expression of $\beta$-Actin. The primers used in this study are listed in Table 1 .

\section{Barrier Function of PLGA/ATT Membranes to Fibroblastic Cells}

Human gingival fibroblasts (HGFs) at passage 4 were applied for barrier function evaluation. Firstly, the Bio Gide membrane, PLGA and PLGA/ATT scaffolds were cut into circular shapes which have the same diameter to the well of a 24-well plate. After irradiation-sterilization, these circles were used to substitute the original membrane at the bottom of the cell culture insert. HGF cells were suspended in Dulbecco's Modified Eagle Medium (DMEM), supplemented with $10 \%(\mathrm{v} / \mathrm{v})$ fetal bovine serum (FBS; Gibco, Carlsbad, CA, USA) at a density of $9.0 \times 10^{3}$ cells $/ \mathrm{mL}$. The cells were seeded into the upper chamber and directly contacted with the surface of the scaffold. After cultured at $37^{\circ} \mathrm{C}$ under a $5 \% \mathrm{CO}_{2}$ atmosphere for 24 hours and 72 hours, the materials were fixed by using 4\% paraformaldehyde (PFA) and stained with 0-6-diamidino-2-phenylindole (DAPI, Sigma-Aldrich). The function of the barrier in the Bio-Gide membrane, PLGA and PLGA/ATT scaffolds was evaluated by

Table I Sequence of Real-Time PCR

\begin{tabular}{|l|l|l|}
\hline Gene & Primer sequence & Length (bp) \\
\hline \multirow{2}{*}{-actin } & F5'-GTAAAGACCTCTATGCCAACA & 21 \\
& R5'- GGACTCATCGTACTCCTGCT & 20 \\
\hline \multirow{2}{*}{ Runx-2 } & F5'- ATCCAGCCACCTTCACTTACAC & 23 \\
& R5'- GGGACCATTGGGAACTGATAGG & 22 \\
\hline \multirow{2}{*}{ ALP } & F5'- TATGTCTGGAACCGCACTGAAC & 22 \\
& R5'- CACTAGCAAGAAGAAGCCTTTGG & 23 \\
\hline \multirow{2}{*}{ OPN } & F5'- CCAAGCGTGGAAACACACAGCC & 22 \\
& R5'- GGCTTTGGAACTCGCCTGACTG & 22 \\
\hline \multirow{2}{*}{ OCN } & F5'- GCCCTGACTGCATTCTGCCTCT & 22 \\
& R5'-TCACCACCTTACTGCCCTCCTG & 22 \\
\hline
\end{tabular}


observing the cell penetration ability under a fluorescence confocal microscope.

\section{Animal Experiments}

Eight beagle dogs (24-week-old, male) received V-shaped buccal dehiscence defects on the distal root of the 3rd and 4th premolars (P3, P4) bilaterally in the mandible under general anesthesia. Briefly, a full-thickness mucoperiosteal flap elevation was performed, and buccal dehiscence defects were created by using a bone chisel. These defects were $5 \mathrm{~mm}$ in height from the cemento-enamel junction (CEJ) and $4 \mathrm{~mm}$ in mesiodistal width at the CEJ. Root surfaces were denuded of the periodontal ligament by using a curette. The flap was repositioned and sutured with absorbable sutures. Further, a dehiscence defect model in canine was considered valid by $\mathrm{CT}$ examination 1 month after the initial surgery. Then, eight dogs were separated into four pairs and allocated randomly into 4 groups for implantation of different materials: group A, negative control (defect without implantation); group B, Bio-Oss bone graft material (Geistlich Biomaterials AG, Wolhusen, Switzerland) covered with the PLGA scaffold; group C, Bio-Oss bone graft material covered with PLGA/ ATT scaffold; group D, Bio-Oss bone graft material covered with Bio-Gide membrane. All scaffolds and membranes used in the surgery were cut into rectangles with the same dimension of $10 \mathrm{~mm} \times 15 \mathrm{~mm}$. The animals received the postoperative antibiotic and analgesic treatment.

\section{Microcomputed Tomography (Micro-CT) Measurement}

The animals were euthanized by using an overdose of sodium pentobarbital $(0.5 \mathrm{~mL} / \mathrm{kg}$, Nembutal injection, Abbott Laboratories, USA) following general anesthesia, 12 weeks after implantation. The fixed specimens were examined by using a microcomputed tomography (microCT) system ( $\mu$ CT-80, Scanco Medical, Switzerland). Moreover, the trabecular number (Tb.N), bone mineral density (BMD), and bone volume fraction (BV/TV) in the bone defect were calculated by using the auxiliary histomorphometric software (Scanco Medical AG, Switzerland).

\section{Sequential fluorescent Labeling}

Polychrome sequential fluorescent labeling for new bone formation and mineralization was performed according to the report of Wu et al. ${ }^{19}$ Briefly, the animals were intraperitoneally injected with $25 \mathrm{mg} / \mathrm{kg}$ tetracycline hydrochloride (TE, Sigma, USA), $30 \mathrm{mg} / \mathrm{kg}$ alizarin red (AL, Sigma, USA), and $20 \mathrm{mg} / \mathrm{kg}$ calcein (CA, Sigma, USA), in the $3 \mathrm{rd}, 6^{\text {th }}$, and 9 th week after the operation, respectively.

\section{Histological and Histomorphometric Observations}

Half of the specimens were dehydrated in alcohol with increasing concentrations from $75 \%$ to $100 \%$ and embedded (undecalcified) in polymethylmethacrylate. Serial sections were prepared from the central aspect of all defect sites along the bucco-lingual direction.

First, the samples were observed for fluorescent labeling under a confocal laser scanning microscope (CLSM; Leica, Germany), and the fluorescent staining corresponding to new bone formation and mineralization was quantified. The pixel numbers with yellow (TE), red (AL), and green $(\mathrm{CA})$ represent the bone regeneration and mineralization 3, 6, and 9 weeks after operation, respectively. Finally, the samples were stained with van Gieson's picrofuchsine for histological observation. The area of newly formed bone was quantified from the serial section collected from each sample, by using a personal computerbased image analysis system (Image Pro Plus 6.0, Media Cybernetic, USA) and reported as the percentage of the whole bone defect area, respectively.

The other specimens from each group were decalcified in 10\% EDTA for 9 months and dehydrated in ethanol before they were embedded in paraffin. Sections were cut into $4 \mu \mathrm{m}$ and stained with the hematoxylin and eosin stain or with Masson's trichrome.

\section{Immunohistochemistry}

For immunohistochemical staining, the decalcified sections were deparaffinized, hydrated with xylene and graded alcohols, and then incubated with primary Collagen-I (Col-I) (Source: goat, Southern Biotech, USA), OPN (Source: rabbit; 1:100 dilution; Novus Biologicals, USA), and OCN antibodies (Source: rat; 1:100 dilution; Abcam, USA). The slides were incubated with horseradish peroxidase (HRP)-labeled secondary antibodies and staining was performed using the 3,3'diaminobenzidine (DAB) substrate. The slides were then counterstained with hematoxylin and mounted. 


\section{Statistical Analysis}

All measurements are expressed as the mean \pm SD. Significant differences between groups were determined using ANOVA (SPSS, v.17.5, USA), while $\mathrm{p}<$ 0.05 was considered to indicate statistical significance.

\section{Results}

\section{Characterization of Electrospun} ATT-Doped PLGA Fibrous Scaffold

In this study, the average of the Bio-Gide membrane was $0.48 \mathrm{~mm}$, the PLAG was $0.33 \mathrm{~mm}$ and the PLGA/ATT was $0.35 \mathrm{~mm}$, respectively (Figure S1, Supporting Information). The morphology of the electrospun fibrous PLGA scaffold (PLGA), ATT-doped PLGA scaffold (PLGA/ATT), and Bio-Gide membrane was observed by SEM (Figure 1A). All the three types of scaffolds displayed a porous and interconnected structure. The range of fiber diameter in the PLGA/ATT scaffold was from 339 to $645 \mathrm{~nm}$. The Bio-Gide membrane is a porous scaffold composed of fibers, and each of the thick fibers (about several micrometers) is surrounded by countless tiny fibers $(100-600 \mathrm{~nm})$. XRD characterization was conducted to determine the crystalline morphology of PLGA and PLGA/ATT composite fibrous scaffolds. As shown in Figure 1B, the XRD diffraction peak (at the $2 \theta$ angle of approximately $21^{\circ}$ ) of the PLGA/ATT composite fibrous scaffolds was broader than that of the neat PLGA fibrous scaffold. The XRD profile of the composite fibers also showed two other diffraction peaks at the $2 \theta$ angles of $8.3^{\circ}$ and $26.5^{\circ}$, which were attributed to the ATT single crystals. $^{20}$ Table 2 shows that the porosity of the composite fibrous mat decreased from $72.1 \%$ to $64.4 \%$ after incorporation of 3\% ATT, resulting in a much denser fiber arrangement. The contact angle of PLGA nanofibers $\left(128.7^{\circ} \pm 3.6^{\circ}\right)$ decreases to $112.6^{\circ} \pm 1.1^{\circ}$ after doping with $3 \%$ ATT (Table 2).

\section{Mechanical Properties}

The stress-strain curves of the PLGA and PLGA/ATT scaffolds and Bio-Gide membrane are showed in Figure 2A. In the PLGA and PLGA/ATT groups, the Young's modulus of elasticity (E) was of no significant difference between the non-immersed samples and the samples immersed for 1 day and 7 days. However, the Young's modulus of elasticity of Bio-Gide presented dramatically decline (Figure 2B). Comparing the three non-immersed materials, the Bio-Gide membrane had the highest value of the ultimate tensile strength (UT). However, after being immersed in 1 day and 7 days, the UT value of Bio-Gide decreased from 5.15 MPa to 2.39 $\mathrm{MPa}$ and $0.90 \mathrm{MPa}$, while the UT value of the PLGA and that of PLGA/ATT scaffold kept stable. The UT value of the PLGA/ATT scaffold was higher than PLGA scaffold attributed to the mixing of ATT particles, reinforcing the scaffold (Figure 2C). The elongation at break revealed that the Bio-Gide membrane was much easier to deform, and the value increased with the cultured time. The PLGA and PLGA/ATT scaffolds presented the similar results of no significant changes between the non-immersed samples and the immersed samples (Figure 2D). The evaluation of these mechanical properties revealed that PLGA/ATT scaffold exhibited better mechanical stability than the Bio-Gide

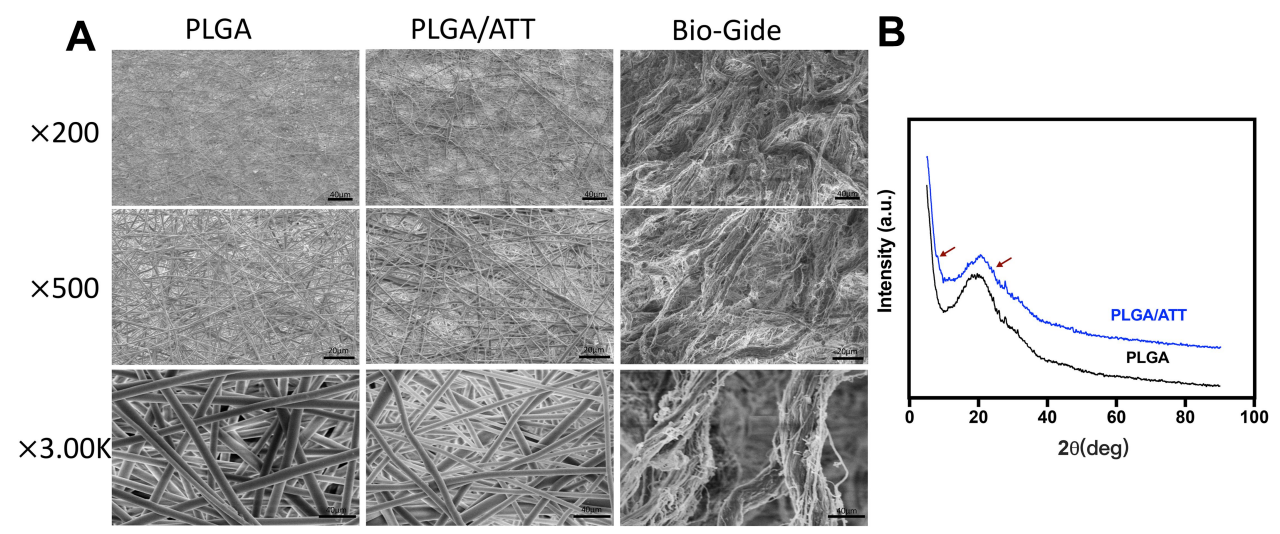

Figure I (A) SEM micrographs of PLGA, PLGA/ATT scaffolds and Bio-Gide membrane (magnification $\times 200, \times 500, \times 3.00 K$ ). (B) XRD patterns of the neat PLGA and PLGA/ATT composite fibrous scaffolds. The red arrow indicates two diffraction peaks that originated from ATT single crystals. Red arrows indicate the XRD diffraction peak of the ATT. Abbreviations: ATT, attapulgite; PLGA, poly(lactic-co-glycolic acid) (PLGA); SEM, scanning electron microscopy; XRD, X-ray diffraction. 
Table 2 Apparent Density, Bulk Density and Porosity of PLGA and ATT-Doped PLGA Electrospun Fibrous Scaffolds (All Data are Given as mean $\pm S D, n=3$ )

\begin{tabular}{|l|l|l|l|l|}
\hline Sample & $\begin{array}{l}\text { Porosity } \\
\text { (\%) }\end{array}$ & $\begin{array}{l}\text { Apparent } \\
\text { Density } \\
\left(\mathbf{g ~ c m}^{\mathbf{3}}\right)\end{array}$ & $\begin{array}{l}\text { Bulk } \\
\text { Density } \\
\left(\mathbf{( ~ c m}^{\mathbf{3}}\right)\end{array}$ & $\begin{array}{l}\text { Water } \\
\text { Contact } \\
\text { Angle (o) }\end{array}$ \\
\hline PLGA & $72.1 \pm 0.1$ & $0.35 \pm 0.04$ & 1.25 & $128.7 \pm 3.6$ \\
PLGA/ATT & $64.4 \pm 0.1$ & $0.45 \pm 0.03$ & 1.27 & $112.6 \pm 1.1$ \\
\hline
\end{tabular}

membrane. ATT enhanced the strength of the PLGA scaffold to optimize the function of the polymer scaffold.

\section{Morphology of Seeded BMSCs and Cell Proliferation Assay}

Due to the rough and uneven thickness of Bio-Gide fiber, it would be difficult to distinguish between tiny fibers and pseudopodia extending by BMSCs in appearance. Furthermore, SEM images at high magnification $(\times 3000)$ were taken for confirmation (Figure S2, Supporting Information). All SEM analysis results demonstrated that the BMSCs attached and spread well on the surface of these scaffolds, revealing that all these membranes exhibited good cell compatibility (Figure 3A-C).
A

\section{Stress-strain curve}

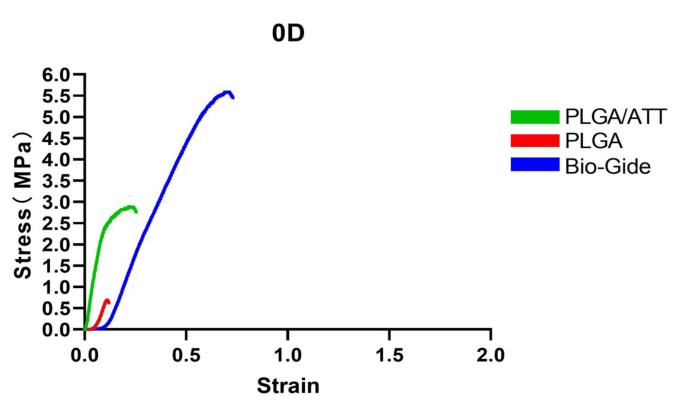

1D

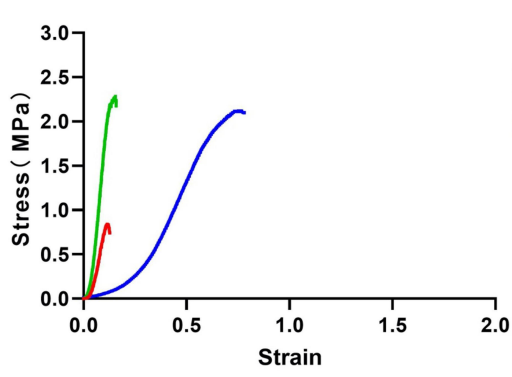

7D

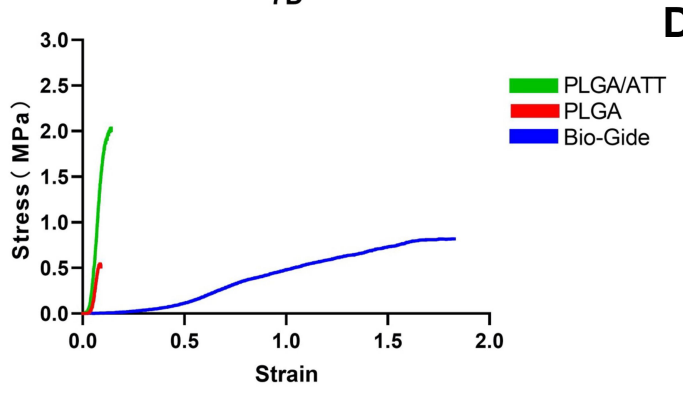

B

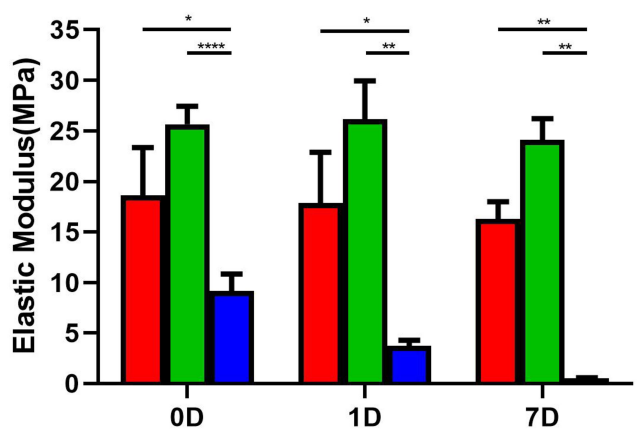

C

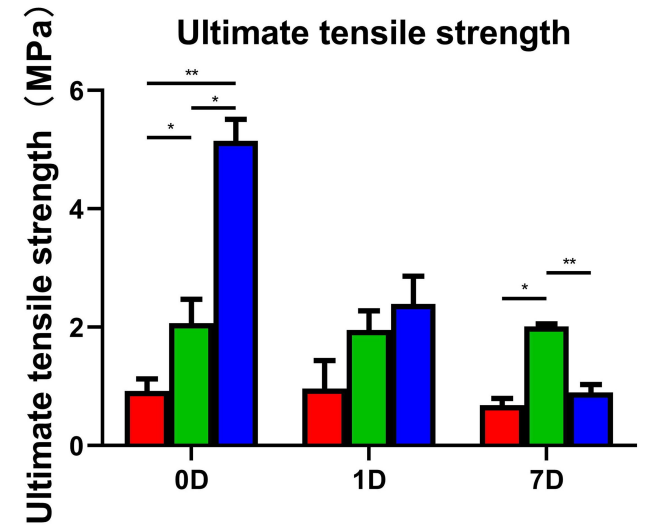

Elongation at break

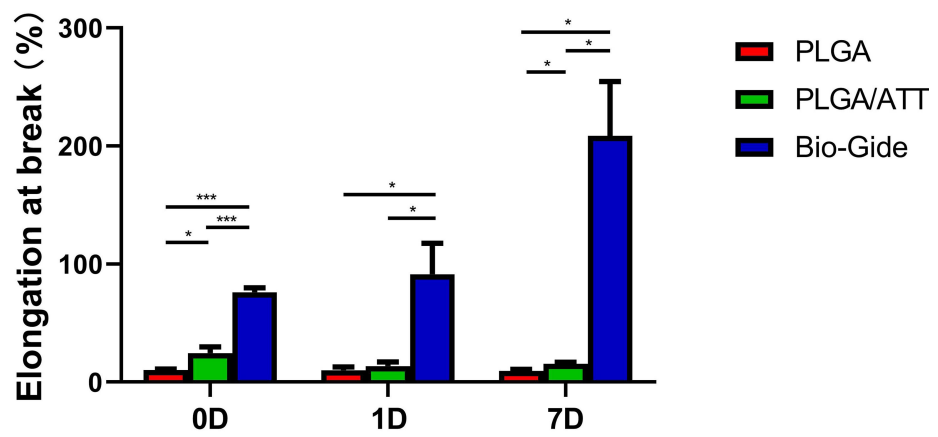

Figure 2 (A) Stress-strain curves of the PLGA, PLGA/ATT scaffolds and the Bio-Gide membrane. (B) E of the PLGA, PLGA/ATT scaffolds and the Bio-Gide membrane. (C) UT value of the PLGA, PLGA/ATT scaffolds and the Bio-Gide membrane. (D) Elongation at break of the PLGA, PLGA/ATT scaffolds and the Bio-Gide membrane. (*P < 0.05). Abbreviations: ATT, attapulgite; PLGA, poly(lactic-co-glycolic acid) (PLGA); E, Young's modulus of elasticity; UT, ultimate tensile strength. 

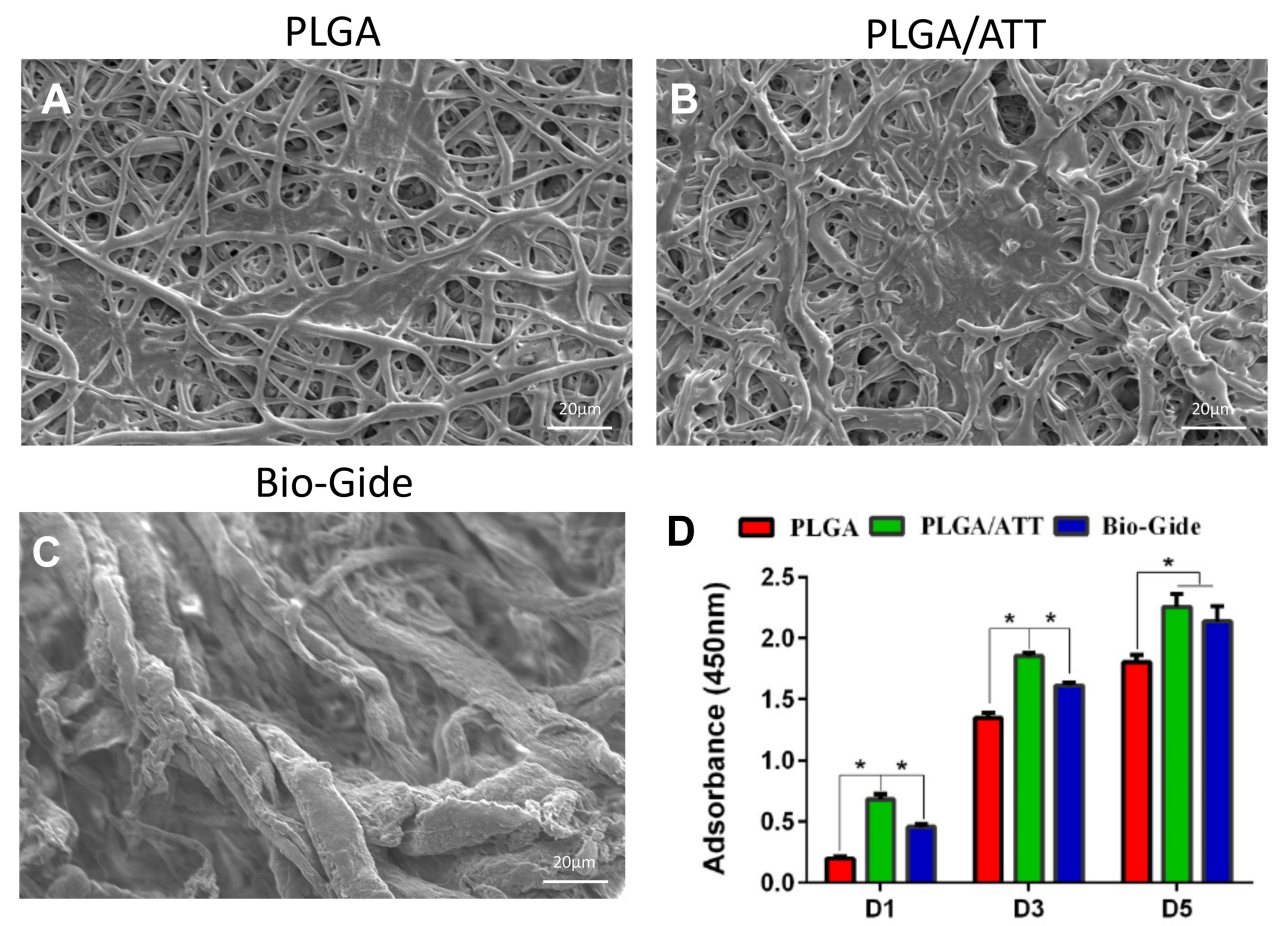

Figure 3 BMSC attachment and proliferation on PLGA, PLGA/ATT and Bio-Gide scaffolds. (A) SEM micrographs of BMSCs attached on three materials (magnification $\times 500$ ). (B) CCK-8 assay of the attachment and proliferation viability of BMSCs cultured on three scaffolds ( $\left.{ }^{\mathrm{p}}<0.05\right)$.

Abbreviations: BMSCs, bone marrow mesenchymal stem cells; PLGA, poly(lactic-co-glycolic acid) (PLGA); ATT, attapulgite; SEM, scanning electron microscopy; CCK8, cell counting kit-8.

As per the results of our previous study, the PLGA/ ATT scaffold demonstrated an excellent ability to induce osteoblastic differentiation of BMSCs. The CCk-8 assay results show that cell proliferation on the PLGA/ATT was better than that on the PLGA on the 1st, 3rd, and 5th days $(P<0.05)$. Moreover, cell proliferation on the surface of the PLGA/ATT scaffold was significantly higher than that on the Bio-Gide on the 1st and 3rd days, while the cell viabilities after 5 days for the PLGA/ATT scaffold and the Bio-Gide membrane were similar (Figure 3D).

\section{ALP Activity Assay and ARS Staining}

The ALP staining was found to be more intense for the BMSCs cultured on the PLGA/ATT scaffold than for those on the PLGA scaffold (Figure 4A). Moreover, the ALPactivity quantitative analysis results show that the ALP activity for the cells cultured on the PLGA/ATT scaffold was higher than that on the PLGA scaffold on the 3rd and 7th days $(\mathrm{p}<0.05)$ (Figure 4B). Round or oval calcified nodules were observed after ARS staining in all groups, and more mineralized nodules were formed in the PLGA/ ATT group, which is similar to that observed for the BioGide group (Figure 4C). Although the ALP activity induced by the PLGA/ATT scaffold was less than that by the Bio-Gide membrane on the 3rd and 7th days, the longterm effect of these two groups on mineralization is relatively similar, indicating that ATT effectively promoted the osteogenic mineralization of BMSCs and inorganic salt deposition.

\section{Quantitative Real-Time PCR Assay}

The quantitative real-time PCR assay of the osteoblastrelated gene was performed for BMSCs cultured on the PLGA scaffold, PLGA/ATT scaffold, and Bio-Gide membrane for 3, 7, and 14 days (Figure 5). The results showed that Runx2 expression in the cells cultured on PLGA/ATT and Bio-Gide was more pronounced than that on the PLGA scaffold on the 3rd, 7th, and 14th days, while no significant difference was observed in the culture between the PLGA/ATT scaffold and Bio-Gide membrane on the 3rd and 14th days. Further, the ALP expression in the case of the PLGA/ATT scaffold and Bio-Gide membrane was higher than that in the case of the PLGA scaffold on the 3rd and 7th days, with the Bio-Gide membrane showing the highest value $(\mathrm{p}<0.05)$. Similar to the ALP expression, the OPN expression was up-regulated for cells cultured on the PLGA/ATT scaffold and Bio-Gide membrane on the 3rd and 7th days. No significant difference was 

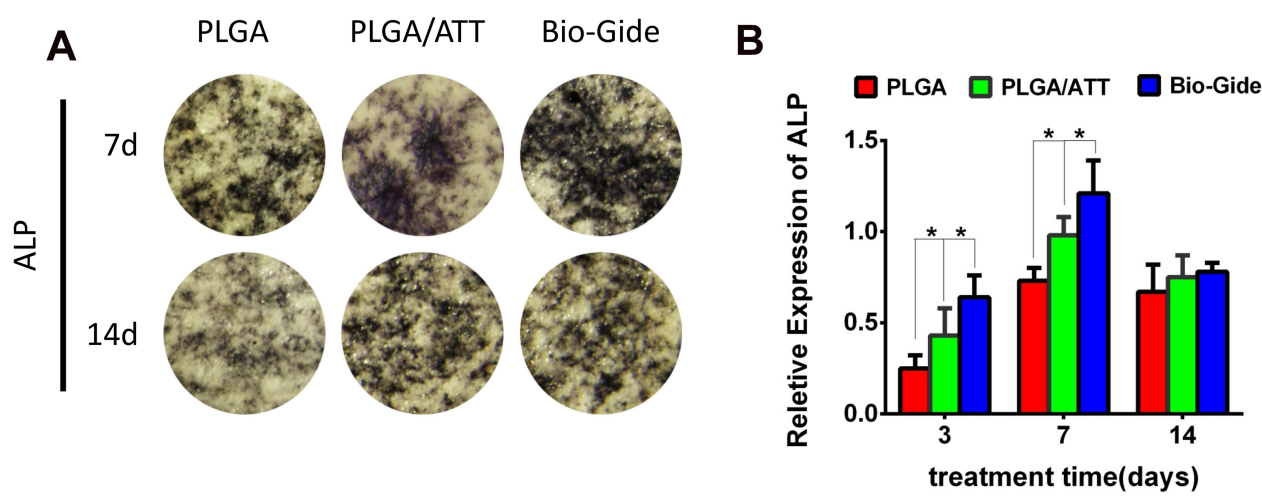

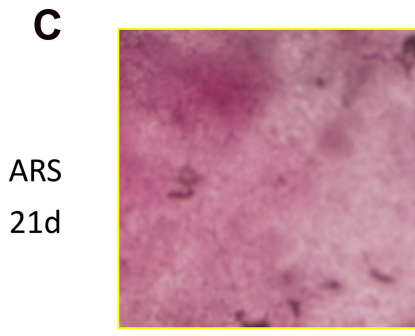

PLGA

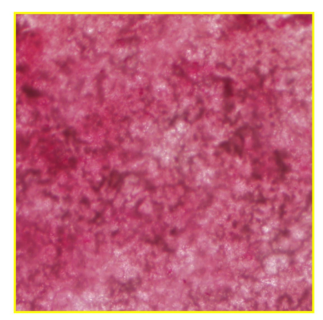

PLGA/ATT

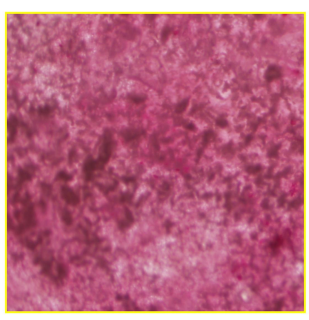

Bio-Gide

Figure 4 Osteogenic differentiation potential of BMSCs seeded on PLGA, PLGA/ATT electrospun scaffold, and Bio-Gide, respectively. (A) ALP staining and (B) quantitative ALP activity (*p < 0.05); (C) ARS staining.

Abbreviations: BMSCs, bone marrow mesenchymal stem cells; PLGA, poly(lactic-co-glycolic acid) (PLGA); ATT, attapulgite; ALP, alkaline phosphatase; ARS, Alizarin red-S.

found in the OCN expression between the PLGA/ATT scaffold and Bio-Gide membrane groups on the 14th day (Figure 5).

\section{Barrier Function}

The physical barrier function of the PLGA/ATT scaffold was tested in vitro by simulating penetration of fibroblast cells into the membranes. Figure $6 \mathrm{~A}-\mathrm{F}$ show plots HGF cells on the surfaces of the membranes at 24 hours and 72 hours. Figure 6A, B, D and E show that no cells penetrate through the opposite side of the PLGA and PLGA/ATT membranes, revealing the scaffolds can prevent HGF cells from penetration. Very few cells, clustering on the opposite side, were found in the Bio-Gide groups. The possible reason was that the double-layered structure of Bio-Gide membrane was not even (Figure 6C and F).

\section{Micro-CT Measurement}

As per the micro-CT results, new bone formation occurred in all groups. From the buccal-lingual view, new bone was formed only at the bottom of the defect in the PLGA scaffold group. The dehiscence defects showed a larger amount of newly formed bone tissue when the PLGA/ATT scaffold or the Bio-Gide membrane were used than when the PLGA scaffold was used 12 weeks after the GBR procedure. Moreover, in the morphometric analysis, significantly greater Tb.N and BMD were detected in the PLGA/ATT group $\left(1.350 \pm 0.0721 \mathrm{~mm}^{-1}\right.$ and $0.512 \pm$ $\left.0.021 \mathrm{~g} / \mathrm{cm}^{3}\right)$ and Bio-Gide group $\left(1.542 \pm 0.2721 \mathrm{~mm}^{-1}\right.$ and $0.553 \pm 0.034 \mathrm{~g} / \mathrm{cm}^{3}$ ) compared with the PLGA group $\left(0.148 \pm 0.012 \mathrm{~mm}^{-1}\right.$ and $\left.0.301 \pm 0.043 \mathrm{~g} / \mathrm{cm}^{3}\right)$; however, no significant difference was found between the PLGA/ ATT group and the Bio-Gide group. Further, a similar outcome was found in these groups in terms of the BV/ TV ratio (Figure 7).

\section{Fluorescent-Labeling Histomorphometric Analysis}

To observe the new bone generation and mineralization, the samples were subjected to fluorescent labeling analysis for 12 weeks. As shown in Figure 8, the percentage of TE labeling (yellow) was $0.63 \pm 0.32 \%, 1.14 \pm 0.42 \%, 2.56 \pm$ $0.36 \%$, and $2.87 \pm 0.43 \%$ for the negative control group, PLGA group, PLGA/ATT group, and Bio-Gide group, respectively. The percentages of the latter two groups are obviously higher than those of the former two groups; further, there was a significant difference in the values between the Bio-Gide and PLGA groups, and between the PLGA/ATT and PLGA groups, but not between the PLGA/ATT and Bio-Gide groups. 


\section{PLGA PLGA/ATT $\square$ Bio-Gide}
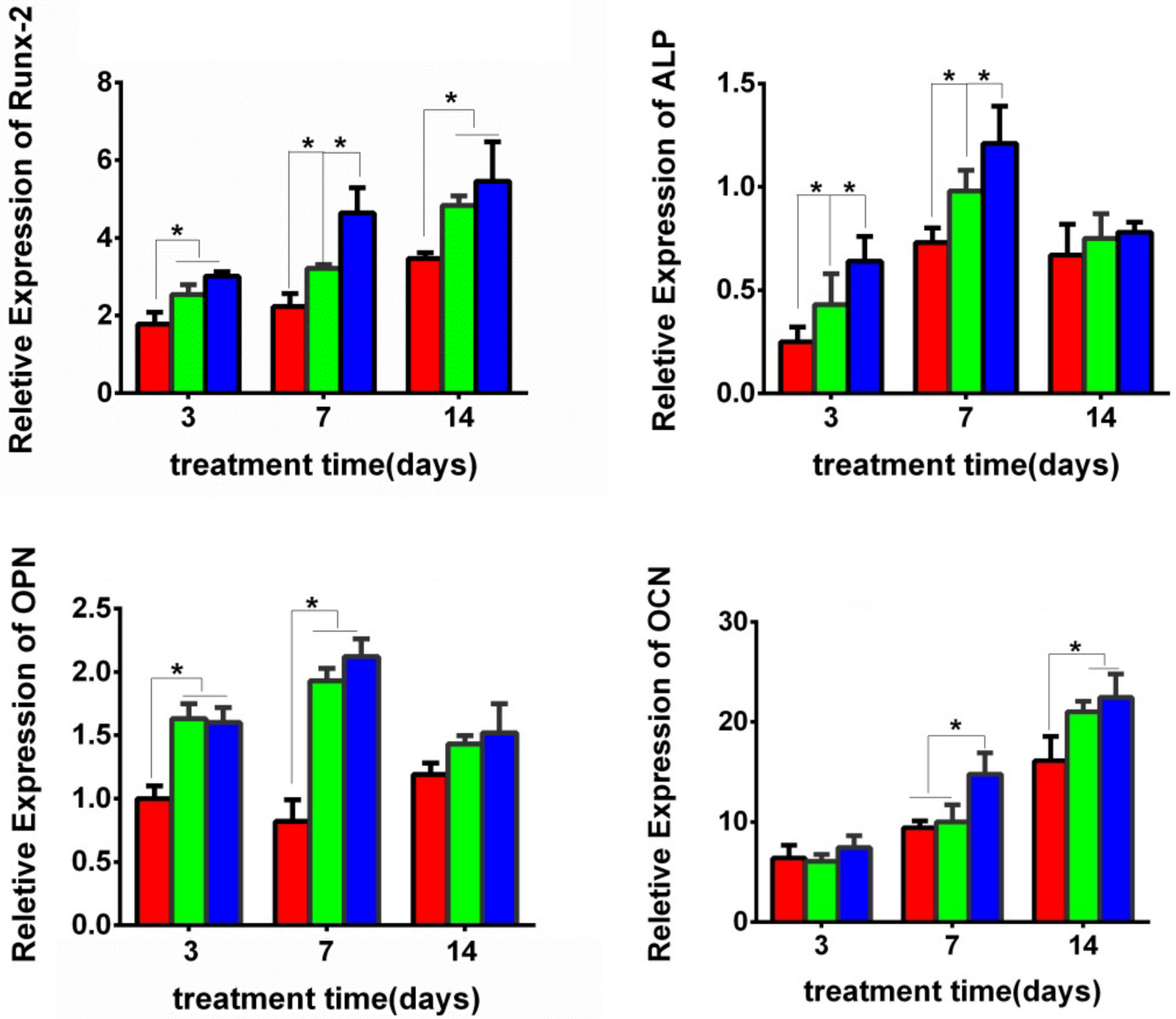

Figure 5 Real-time PCR analysis of Runx-2, ALP, OPN, and OCN mRNA relative expression ( $\left.{ }^{*} \mathrm{P}<0.05\right)$.

Abbreviations: PCR, polymerase chain reaction; Runx2, runt-related transcription factor 2; ALP, alkaline phosphatase; OPN, osteopontin; OCN, osteocalcin.

In the 6th week, the percentage of AL labeling (red) in the PLGA/ATT $(2.64 \pm 0.48 \%)$ and Bio-Gide groups $(2.79 \pm 0.26 \%)$ were obviously higher than that in the PLGA group $(0.89 \pm 0.24 \%)$ and in the negative control group $(0.71 \pm 0.34 \%)$, while there was no significant difference between the PLGA and negative control groups. At week 9, the higher percentage of CA labeling (green) was detected in the PLGA/ATT $(3.58 \pm 0.31)$ and the Bio-Gide group $(3.4 \pm 0.17)$ as compared with the PLGA group (1.45 0.36$)$ $(P<0.05)$ (Figure 8). Taken together, these results demonstrate that, like the Bio-Gide membrane, the PLGA/ATT scaffold can effectively promote new bone formation.

\section{Histological Analysis of Bone}

\section{Regeneration}

Undecalcified specimens stained with van Gieson's picrofuchsin showed that there was a small amount of new bone formation commencing from the bottom of the dehiscence in the negative control group $(10.42 \pm 1.36 \%) 12$ weeks after the operation. However, a greater amount of newly 


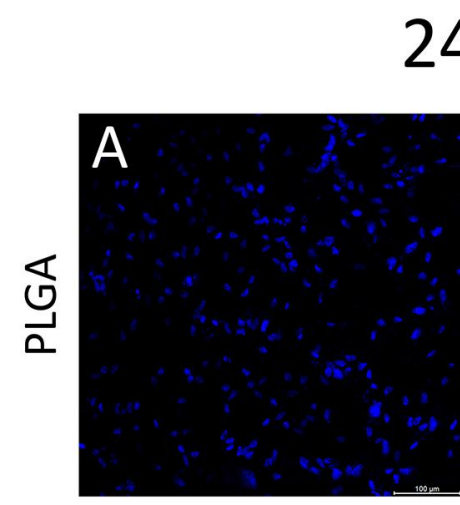

\section{$24 \mathrm{H}$}
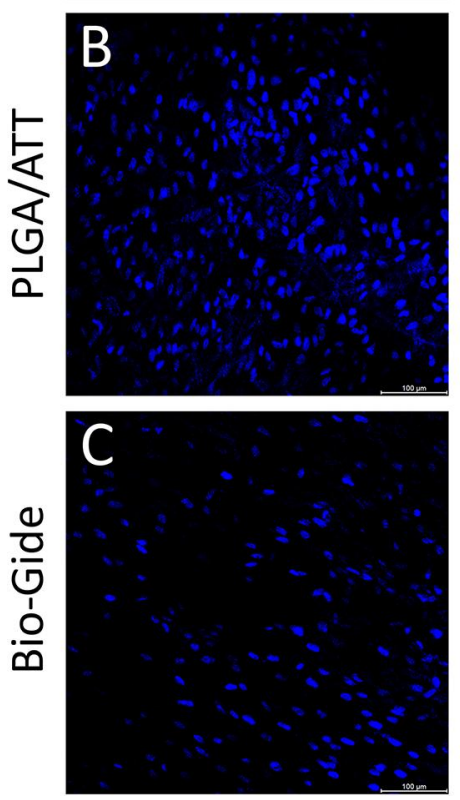
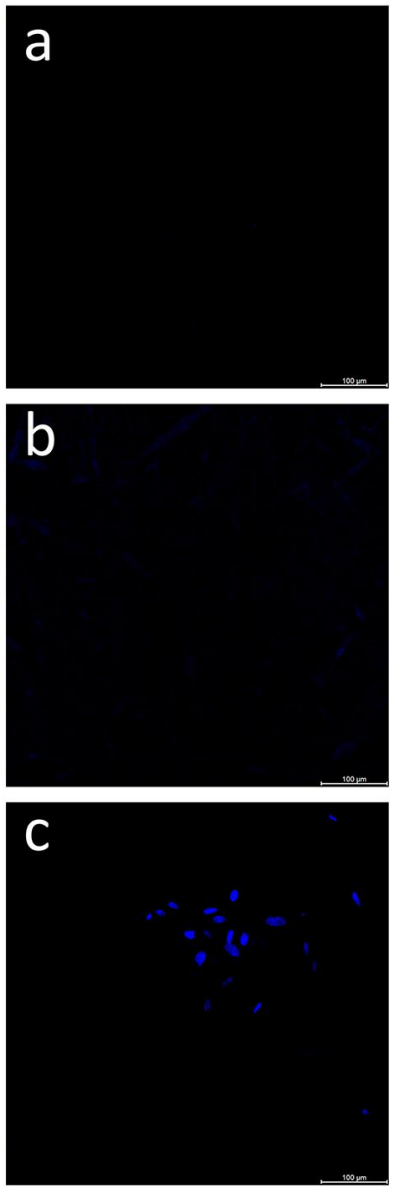

$72 \mathrm{H}$
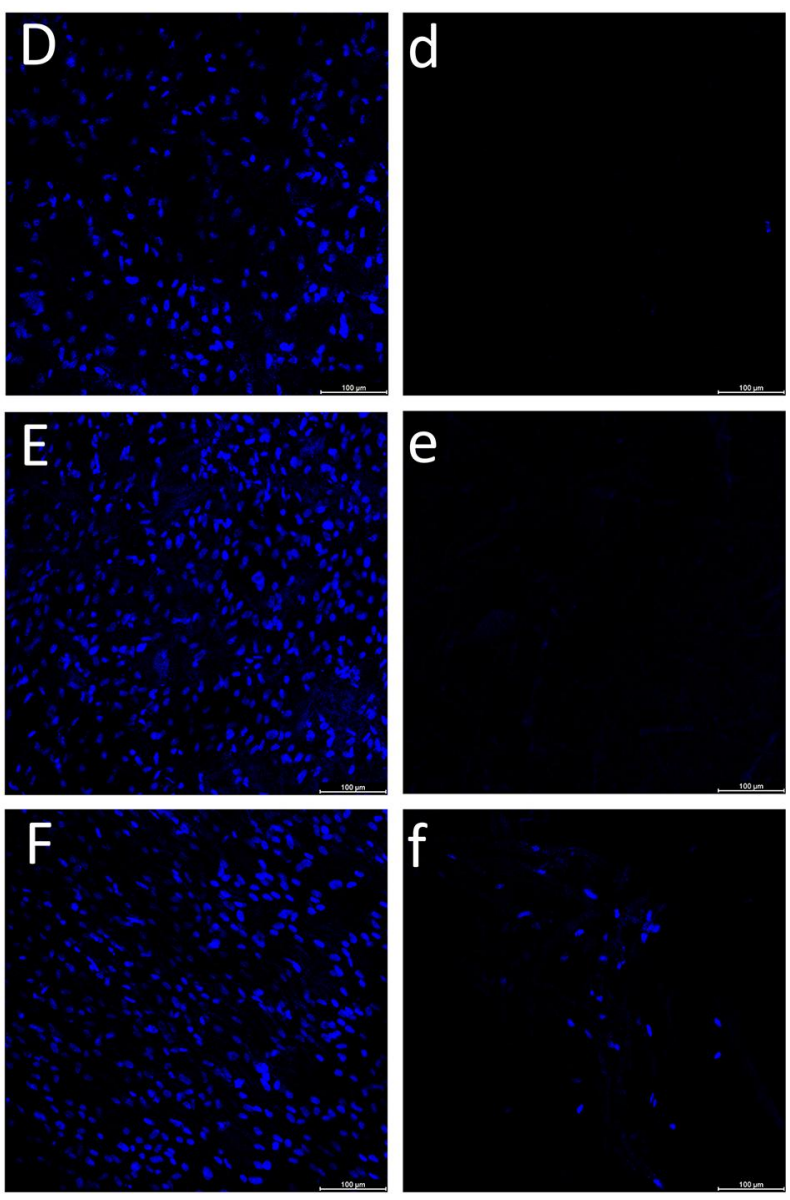

Figure 6 The penetration of HGFs at $24 \mathrm{~h}$ and $72 \mathrm{~h}$. (A)-(C), (D)-(F) show the cells seeded on the surface. (A)-(C), (D)-(F) show the opposite side of the surface. Abbreviation: HGFs, human gingival fibroblasts.

formed bone tissue was detected in the other three groups, especially in the PLGA/ATT group $(27.97 \pm 3.55 \%)$ and Bio-Gide group $(30.56 \pm 1.34 \%)$, with new bone formation observed around remnants of the Bio-Oss. Furthermore, the newly formed bone area in the PLGA/ ATT and Bio-Gide groups was significantly greater than that in the other two groups $(p<0.05)$ (Figure 9).

HE staining of the dehiscence defects in each group in the 12 postoperative weeks showed that the PLGA/ ATT group displayed more obvious new bone formation, abundant thick bone trabeculae, and significant newly formed capillary vessels in the bone graft area. These results demonstrate that the PLGA/ATT scaffold evidently accelerated new bone formation. Further, compared with the other treated groups, a higher amount of residual materials was observed in the PLGA group. However, the defect was filled with abundant fibrous connective tissue in the negative control group (Figure 10, Figure S3 Supporting information).

\section{Immunohistochemistry}

Col-1, OPN, and OCN are considered to be important markers for osteoblast maturation. In addition to RT-PCR gene detection, the immunohistochemical analysis was also performed on deparaffinized sections. High Col-I expression was observed around the newly formed trabecular bone in the PLGA group and in PLGA/ATT group, but not in the negative control group. By contrast, no obvious positive Col-I staining was observed in other groups. Positive brown staining of OPN and OCN was observed evenly in a large area in the newly formed bone in the PLGA/ATT and Bio-Gide groups, with even blood vessels observed in the PLGA/ATT group. The immunohistochemical analysis results thus showed clear evidence 
A

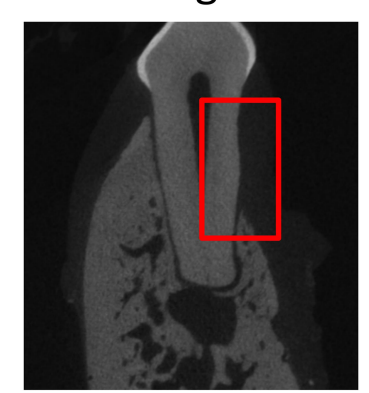

Negative Control

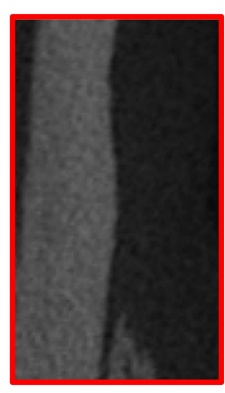

PLGA/ATT
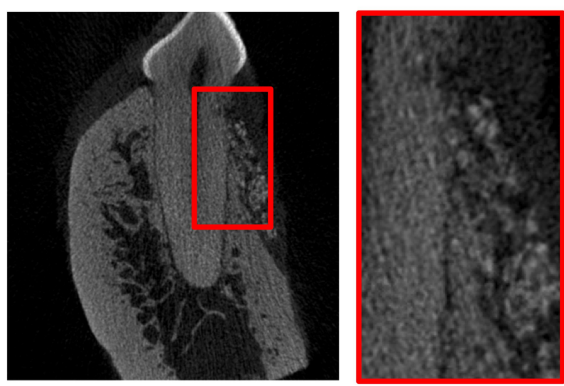

PLGA
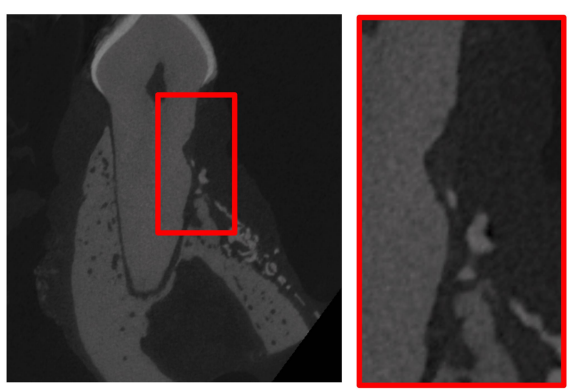

Bio-Gide
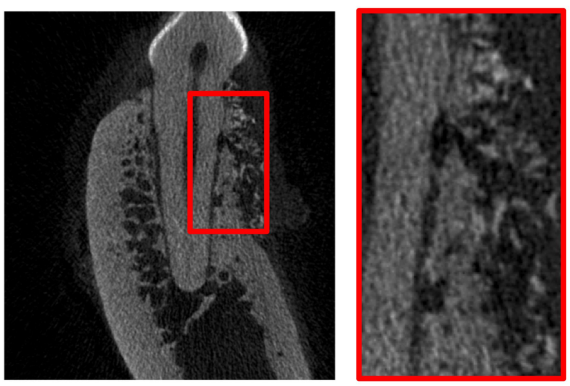

B
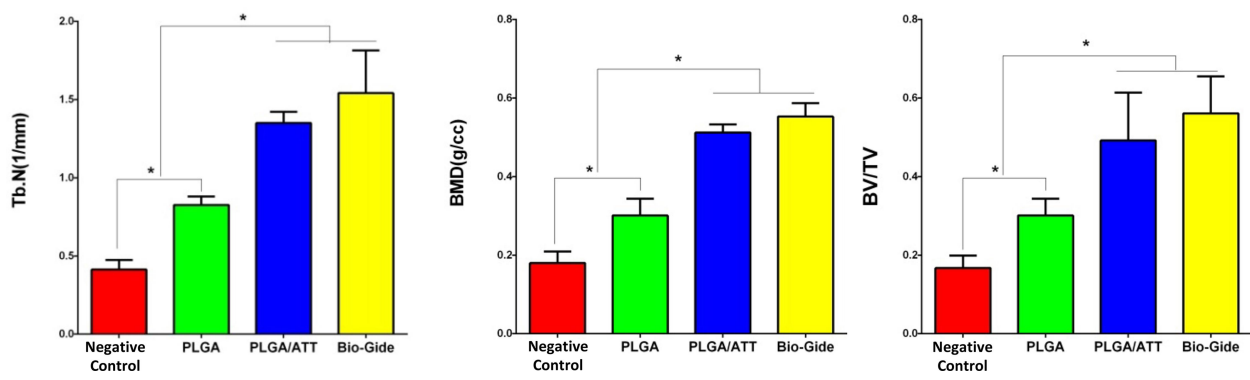

Figure 7 Micro-CT evaluations of the bone defect area. (A) Representative buccal-lingual section of buccal dehiscence defects 12 weeks post-implantation. (B) Morphometric analysis of Tb.N, BMD and BV/TV $(* \mathrm{p}<0.05)$.

Abbreviations: Micro-CT, micro-computed tomography; Tb.N, trabecular number; BMD, bone mineral density; BV/TV, bone volume/total volume.

that the PLGA/ATT scaffold could provide an environment similar to that provided by the Bio-Gide membrane for the maturation of the osteoblasts (Figure 11).

\section{Discussion}

If there is a breach in the continuity of the cortical plate, dehiscence and fenestration may result. Alveolar bone deficiency often leads to less support and resistance to occlusal loading. Thus, these alveolar defects profoundly affect the prognosis of both periodontal, orthodontic, and endodontic treatment outcomes; therefore, they should be carefully managed to improve the long-term prognosis of the tooth.

GBR technology is commonly used for alveolar bone regeneration therapy, in which a membrane not only performs the barrier function to avoid the interference of fibroblasts or epithelial cells but also promotes faster bone growth in clinical applications. ${ }^{21}$ Absorbable barriers for GBR exhibit biocompatibility, and do not need to be removed, which reduces patients' discomfort and eliminates surgery-related complications. The collagen membrane has been most widely used in clinical practice, but its high price inhibits its application. Since the PLGA membrane can be provided for patients at a low cost, its clinical application should be encouraged. The PLGA does not pose the risk of immune responses; ${ }^{16}$ However, BioGide collagen membranes originate from animal sources, which can cause difficulties in terms of patient acceptance and immune responses, and the transmission of infectious agents can never be completely prevented.

ATT particles can be efficiently entrapped in the PLGA nanofibers with no visible ATT crystal after 

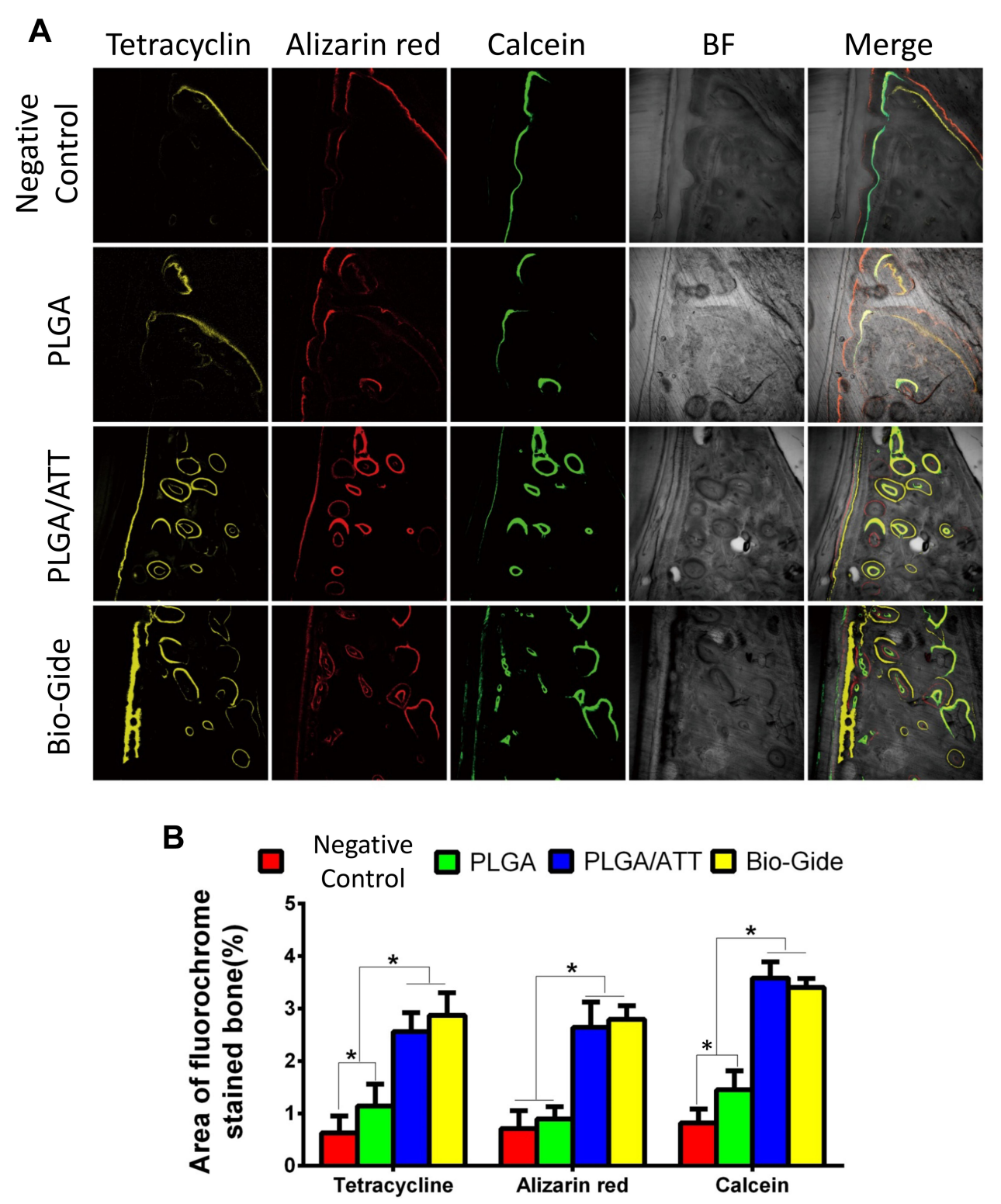

Figure $8 \mathrm{New}$ bone formation and mineralization were determined histomorphometrically by TE, AL, and CA-labeling analysis. (A) Sequential fluorescent labeling of TE, $\mathrm{AL}$, and CA for the negative control, PLGA, PLGA/ATT, and Bio-Gide groups 3, 6, and 9 weeks after implantation. Merged images of the three fluorochromes for the same group. (B) The graph shows the percentage of TE, AL, and CA staining determined by histomorphometric analysis for the four different groups. (*P $<0.05$ ).

Abbreviations: TE, tetracycline hydrochloride; AL, alizarin red; CA, calcein; PLGA, poly(lactic-co-glycolic acid) (PLGA); ATT, attapulgite.

electrospinning. With the spacing among the aggregated single crystals, ATT could be easy to form uniform distribution in the mixture. The XRD results show that the average size of PLGA crystallites in the composite fibers was smaller than that of neat PLGA fibers, since the uniform distribution of nano-sized ATT single crystals could confine the growth of PLGA crystallites in the composite fibers. In addition, the surface hydrophilicity of fibrous scaffold is one of the key parameters affecting their interactions with cells. The hydrophilicity of the PLGA fibrous membrane improved with ATT incorporation. The increased hydrophilicity of PLGA fibers after doping of ATT may facilitate the infiltration of hydrophilic nutrient substances and tune the cell response on the membrane surface.

Through mechanical measurements, it is possible to compare different bio-membranes subject to the same test conditions. Sufficient mechanical strength should be present to bear mechanical loading without membrane collapse. ${ }^{22}$ Although fibers of Bo-Gide are rearranged during tearing due to its deformability and the ensuing orientation increases tear strength, the 
A
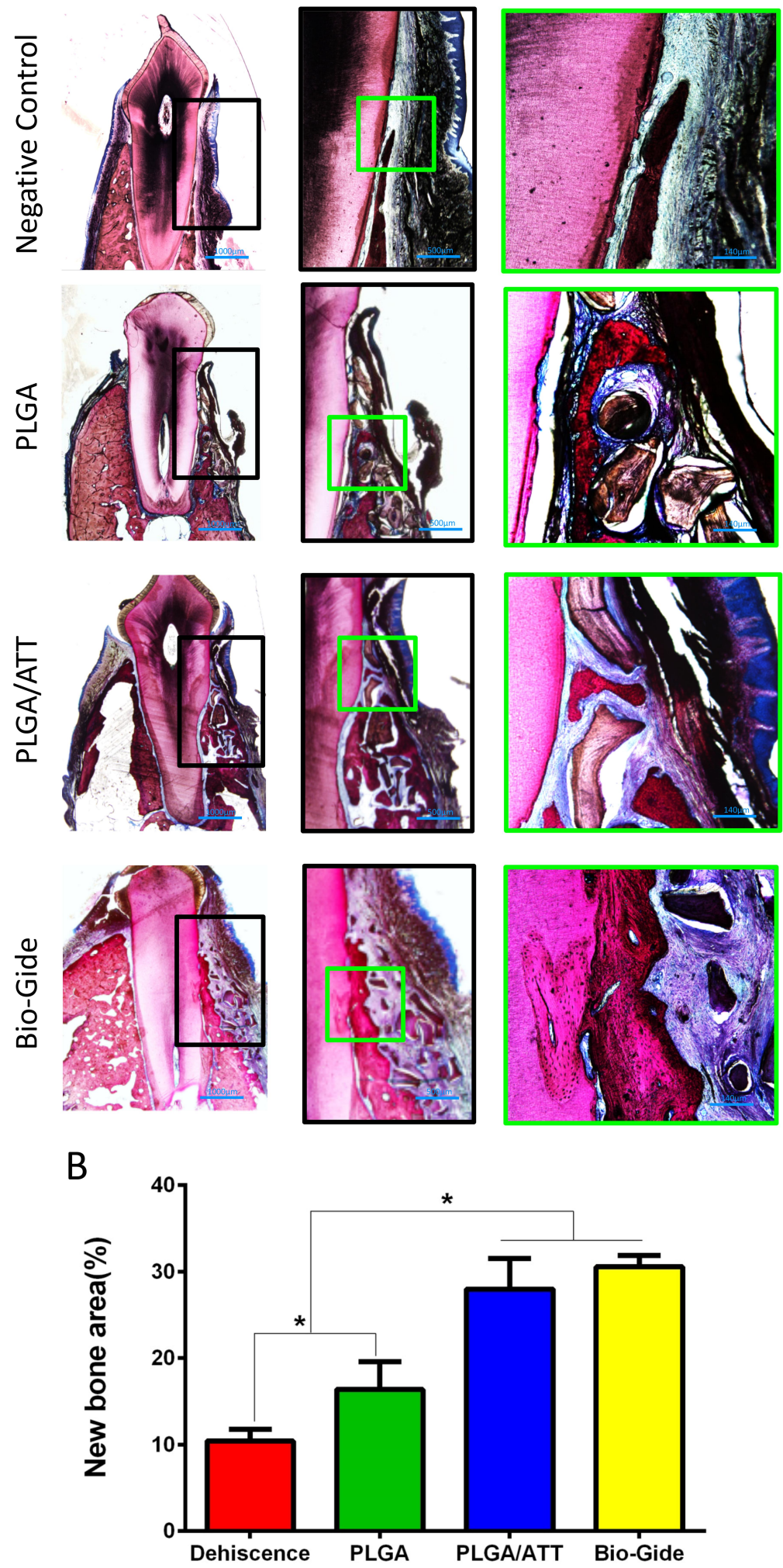

Figure 9 Histological analysis of newly formed bone in buccal dehiscence defects 12 weeks post-implantation. (A) Non-decalcified specimens were sliced, and sections were stained with van Gieson's picrofuchsin (Row I, × 12.5; Row 2, ×40; Row 3, × 100). Red areas represent newly formed bone, blue areas indicate collagen fibers, and black areas represent undegraded remnants. (B) Percentage of new bone area $(* p<0.05)$. 

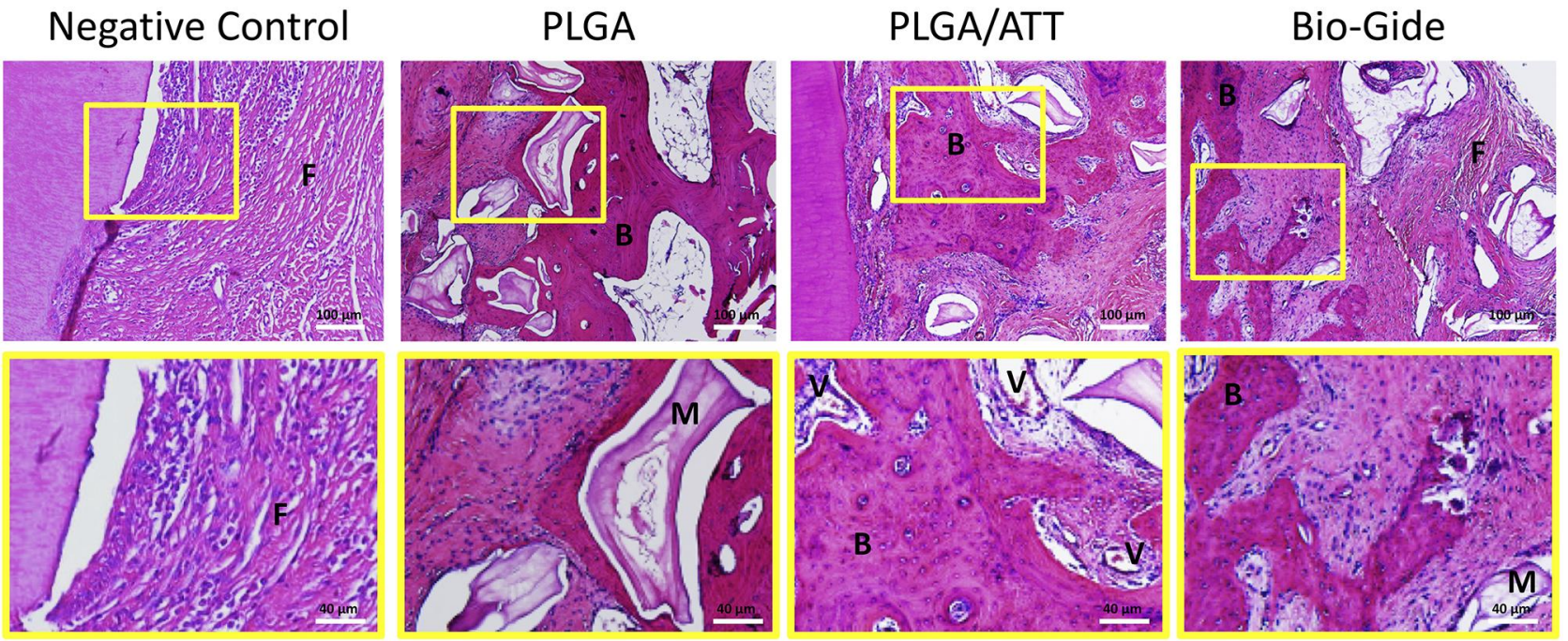

Figure 10 Decalcified HE staining sections of the bone defect area in each group 12 weeks post-implantation. $M$ indicates the residual materials; $\mathrm{V}$, capillary vessels; B, thick bone trabeculae; $M$, residual materials; and $F$, fibrous tissue. Lower magnification images $(\times 100)$ are shown in the upper panel. The region of interest is indicated by the yellow box and its magnified image $(\times 250)$ is presented in the lower panel.

Abbreviations: HE, hematoxylin and eosin; PLGA, poly (lactic-co-glycolic acid) (PLGA); ATT, attapulgite.

Negative Control
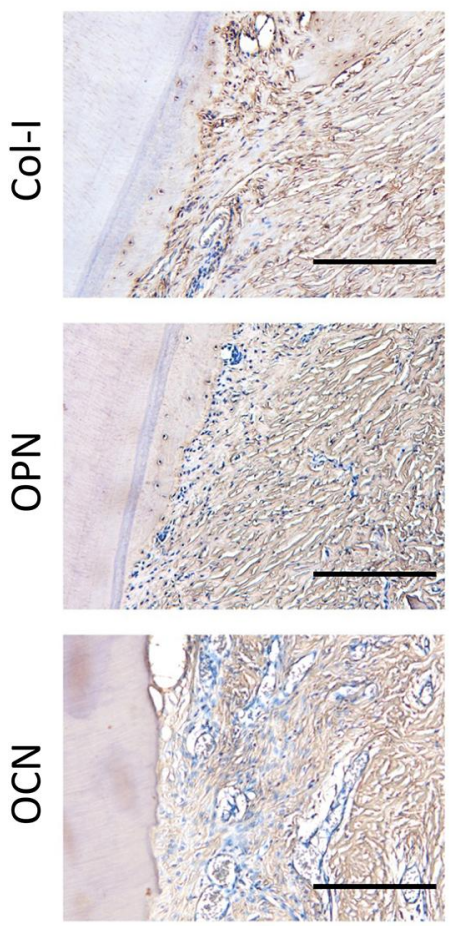

PLGA
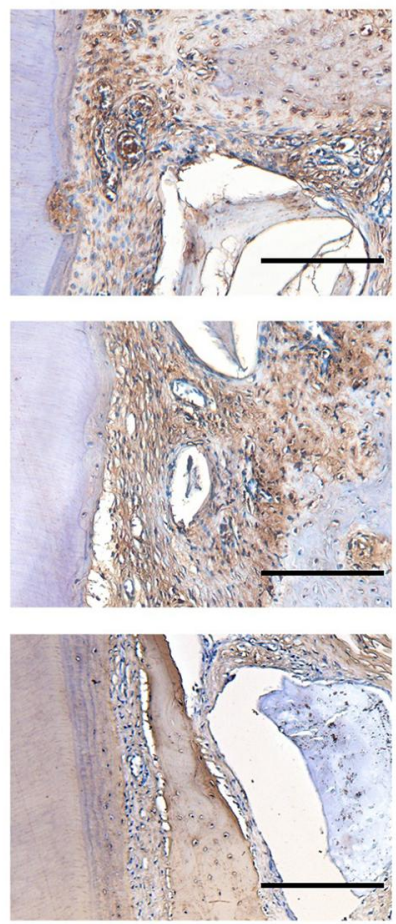

PLGA/ATT
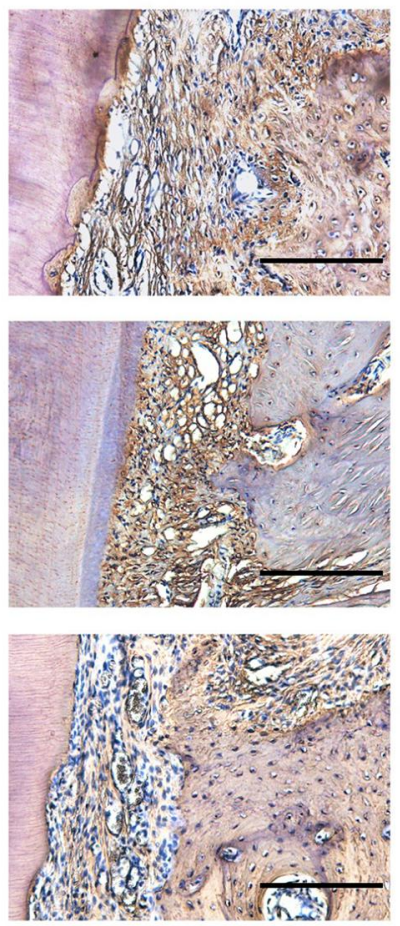

Bio-Gide
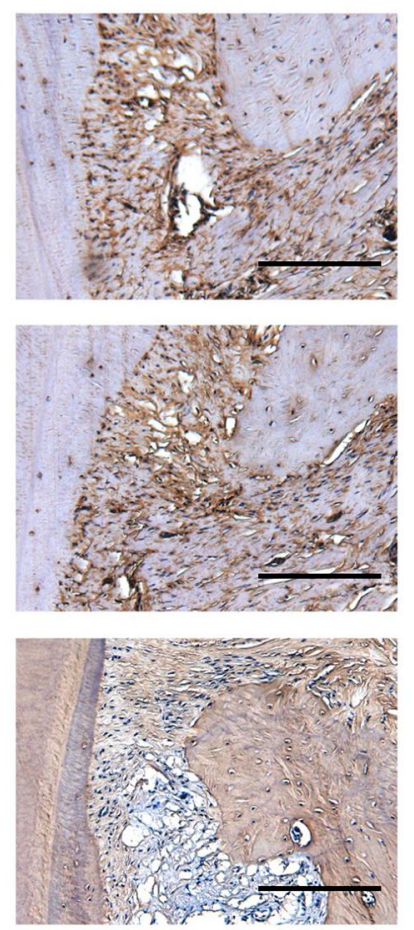

Figure I I Immunohistochemical analysis of new bone formation in each group 12 weeks post-implantation. Immunostaining of Col-I, OPN, and OCN demonstrated more intense brown staining in the bone and the surrounding fibroblastic-like tissue in the PLGA/ATT and Bio-Gide groups (scale bar $=100 \mu \mathrm{m}$ ).

Abbreviations: Col-I, collagen-I; OPN, osteopontin; OCN, osteocalcin; PLGA, poly (lactic-co-glycolic acid) (PLGA); ATT, attapulgite.

ultimate properties declined severely after 7 dayimmersion with PBS. Instead, the PLGA/ATT exhibited the best mechanical performance compared with the other two groups and the ATT was a major contributor to the mechanical strength. The primary structural units of ATT are silicate single crystals that possess a high degree of structural perfection and concomitant superior mechanical properties. 
In addition, ATT crystals have a special threedimensional nanostructure that is responsible for their adsorption capability, which is likely to improve protein adsorption on the scaffold. An ideal scaffold should allow more protein to aggregate on its surface and thus provides better biocompatibility for cell adhesion and proliferation. Another possible mechanism is that clay nanoparticles act as focal adhesion sites, and facilitate cell attachment and spreading by providing reactive functional groups (eg, $\left.>\mathrm{Si}-\mathrm{OH}_{2}{ }^{+}\right) .{ }^{23}$ We found that ALP activity, calcium deposition, and bone-related gene expression for the PLGA/ATT scaffolds were comparable to those of the Bio-Gide membrane, indicating a directly bioactive effect of ATT on the osteogenic differentiation of skeletal stem cell populations. A priority for future studies is to investigate the potential mechanisms by which clay promotes osteogenic differentiation.

A synthetic polymer membrane does not have osteoinduction ability without incorporating bio-active molecules. ${ }^{24} \mathrm{Si}$, a vital element in metabolism, collagen synthesis and bone mineralization, plays an essential role in skeletal development. ${ }^{25}$ Similarly, $\mathrm{Mg}$ is essential for bone tissue mineralization. ${ }^{26}$ Therefore, ATT, an Mg- and Si-containing clay material, may have the osteogenic ability for bone regeneration. Our in vivo experiment showed that the PLGA/ATT group could promote the newly formed bone tissue penetrated into transplantation bones, which was nearly reached the levels of the Bio-Gide group. This was observed by micro-CT, sequential fluorescent labeling, histological observation, and immunohistochemistry analyses. Based on these results, the PLGA/ ATT scaffold was considered to have the ability to promote alveolar bone regeneration in the defect area, which was comparable with the Bio-Gide membrane, suggesting that the PLGA/ATT scaffold is promising for bone tissue engineering applications.

Previous researches reported that the incorporation of inorganic materials would cause a reduction in porosity. ${ }^{12,27}$ The porosity of biomaterials plays a vital role in bone formation, and the appropriate porosity would enhance the progression of osteogenesis. ${ }^{28,29} \mathrm{In}$ the future, we will further investigate the relationship between the porosity and mechanical strength of the PLGA/ATT composite nanofibers used for bone regeneration, which will enable the fabrication of scaffolds with morphological and mechanical properties needed for bone repair with a particular design.

\section{Conclusion}

In this study, we demonstrated that the electrospun ATTdoped PLGA fibrous scaffold exhibited excellent fibroblastic barrier function and favorable osteogenic effects. In the dehiscence model, the effect of PLGA/ATT scaffold on bone regeneration was similar to that of the Bio-Gide membrane. With the widely available raw materials, low cost, and simple process, electrospun ATT-doped PLGA fibrous scaffolds are promising economic membrane barriers for repairing bone defects.

\section{Acknowledgments}

This study was supported by the National Natural Science Foundation of China (81800932, 31600777), Project of Outstanding Youth Backbone of the 9th Hospital (jyyq08201601), and Research Fund of Medicine and Engineering of Shanghai Jiao Tong University (YG2016QN04). Clinical Research Project of MultiDisciplinary Team, Shanghai Ninth People's Hospital, Shanghai Jiao Tong University School of Medicine (201913). Xinru Xie and Xiangyang Shi are co-first authors for this study.

\section{Author Contributions}

All authors contributed towards data analysis, drafting, and critical revision of the paper, gave final approval of the version to be published, and agreed to be held accountable for all aspects of the work.

\section{Disclosure}

The authors report no conflicts of interest with respect to the authorship and/or publication of this article.

\section{References}

1. Enhos S, Uysal T, Yagci A, Veli I, Ucar FI, Ozer T. Dehiscence and fenestration in patients with different vertical growth patterns assessed with cone-beam computed tomography. Angle Orthod. 2012;82 (5):868-874. doi:10.2319/111211-702.1

2. Leung CC, Palomo L, Griffith R, Hans MG. Accuracy and reliability of cone-beam computed tomography for measuring alveolar bone height and detecting bony dehiscences and fenestrations. $\mathrm{Am}$ J Orthod Dentofacial Orthop. 2010;137(4):S109-19. doi:10.1016/j. ajodo.2009.07.013

3. Sun L, Zhang L, Shen G, Wang B, Fang B. Accuracy of cone-beam computed tomography in detecting alveolar bone dehiscences and fenestrations. Am J Orthod Dentofacial Orthop. 2015;147(3):31 3-323. doi:10.1016/j.ajodo.2014.10.032

4. Lee KM, Kim YI, Park SB, Son WS. Alveolar bone loss around lower incisors during surgical orthodontic treatment in mandibular prognathism. Angle Orthod. 2012;82(4):637-644. doi:10.2319/08171 $1-526.1$ 
5. Kim Y, Park JU, Kook YA. Alveolar bone loss around incisors in surgical skeletal Class III patients. Angle Orthod. 2009;79 (4):676-682. doi:10.2319/070308-341.1

6. Chiapasco M, Romeo E, Casentini P, Rimondini L. Alveolar distraction osteogenesis vs. vertical guided bone regeneration for the correction of vertically deficient edentulous ridges: a 1-3-year prospective study on humans. Clin Oral Implants Res. 2004;15(1):82-95. doi:10.1111/j.1600-0501.2004.00999.x

7. Sudarsan S, Arun KV, Priya MS, Arun R. Clinical and histological evaluation of alloderm GBR and BioOss in the treatment of Siebert's class I ridge deficiency. J Indian Soc Periodontol. 2008;12(3):73-78. doi:10.4103/0972-124X.44099

8. Behring J, Junker R, Walboomers XF, Chessnut B, Jansen JA. Toward guided tissue and bone regeneration: morphology, attachment, proliferation, and migration of cells cultured on collagen barrier membranes. Sys Rev Odontol. 2008;96:1-11.

9. Leal AI, Caridade SG, Ma J, et al. Asymmetric PDLLA membranes containing Bioglass $(\mathrm{R})$ for guided tissue regeneration: characterization and in vitro biological behavior. Dent Mater. 2013;29 (4):427-436. doi:10.1016/j.dental.2013.01.009

10. Rakhmatia YD, Ayukawa Y, Furuhashi A, Koyano K. Current barrier membranes: titanium mesh and other membranes for guided bone regeneration in dental applications. J Prosthodont Res. 2013;57 (1):3-14. doi:10.1016/j.jpor.2012.12.001

11. Lee SW, Kim SG. Membranes for the Guided Bone Regeneration. Maxillofac Plast Reconstr Surg. 2014;36(6):239-246. doi:10.14402/ jkamprs.2014.36.6.239

12. Wang Z, Zhao Y, Luo Y, et al. Attapulgite-doped electrospun poly (lactic-co-glycolic acid) nanofibers enable enhanced osteogenic differentiation of human mesenchymal stem cells. RSC Adv. 2014;5 (4):2383-2391. doi:10.1039/C4RA09839K

13. Amoroso NJ, D’Amore A, Hong Y, Sacks MS, Wagner WR. Elastomeric electrospun polyurethane scaffolds: the interrelationship between fabrication conditions, fiber topology, and mechanical properties. Adv Mater. 2011;23(1):106-111. doi:10.1002/adma.201003210

14. Ong BYS, Ranganath SH, Lai YL, et al. Paclitaxel delivery from PLGA foams for controlled release in post-surgical chemotherapy against glioblastoma multiforme. Biomaterials. 2009;30(18):3189. doi:10.1016/j.biomaterials.2009.02.030

15. Lee JY, Bashur CA, Goldstein AS, Schmidt CE. Polypyrrole-coated electrospun PLGA nanofibers for neural tissue applications. Biomaterials. 2009;30(26):4325. doi:10.1016/j.biomaterials.2009.04.042

16. Jung RE, Kokovic V, Jurisic M, Yaman D, Subramani K, Weber FE. Guided bone regeneration with a synthetic biodegradable membrane: a comparative study in dogs. Clin Oral Implants Res. 2011;22 (8):802-807. doi:10.1111/j.1600-0501.2010.02068.x

17. Xu L, Jiang L, Xiong C, Jiang L, Ye L. Study on a novel doublelayered composite membrane of $\mathrm{Mg}$-substituted nano-hydroxyapatite /poly(1 -lactide-co- $\varepsilon$-caprolactone): effect of different 1 -lactide/ $\varepsilon$ caprolactone ratios. Mater Sci Eng A. 2014;615:361-366. doi:10.10 16/j.msea.2014.07.044

International Journal of Nanomedicine

\section{Publish your work in this journal}

The International Journal of Nanomedicine is an international, peerreviewed journal focusing on the application of nanotechnology in diagnostics, therapeutics, and drug delivery systems throughout the biomedical field. This journal is indexed on PubMed Central, MedLine, CAS, SciSearch ${ }^{\mathbb{R}}$, Current Contents ${ }^{\mathbb{B}} /$ Clinical Medicine,
18. Tian M, Gao Y, Liu Y, et al. Bis-GMA/TEGDMA Dental Composites Reinforced with Electrospun Nylon 6 Nanocomposite Nanofibers Containing Highly Aligned Fibrillar Silicate Single Crystals. Polymer. 2007;48(9):2720-2728. doi:10.1016/j.polymer. 2007.03.032

19. Wu Y, Cao L, Xia L, et al. Evaluation of Osteogenesis and Angiogenesis of Icariin in Local Controlled Release and Systemic Delivery for Calvarial Defect in Ovariectomized Rats. Sci Rep. 2017;7(1):5077. doi:10.1038/s41598-017-05392-z

20. Tian M, Qu C, Feng Y, Zhang L. Structure and properties of fibrillar silicate/SBR composites by direct blend process. $J$ Mater Sci. 2003;38(24):4917-4924. doi:10.1023/B:JMSC.0000004414.27 574.93

21. Geurs NC, Korostoff JM, Vassilopoulos PJ, et al. Clinical and histologic assessment of lateral alveolar ridge augmentation using a synthetic long-term bioabsorbable membrane and an allograft. J Periodontol. 2008;79(7):1133-1140. doi:10.1902/jop.2008.070 595

22. Bottino MC, Thomas V, Janowski GM. A novel spatially designed and functionally graded electrospun membrane for periodontal regeneration. Acta Biomater. 2011;7(1):216-224. doi:10.1016/j. actbio.2010.08.019

23. Gaharwar AK, Kishore V, Rivera C, et al. Physically crosslinked nanocomposites from silicate-crosslinked PEO: mechanical properties and osteogenic differentiation of human mesenchymal stem cells. Macromol Biosci. 2012;12(6):779-793. doi:10.1002/mabi.201100508

24. Jones AA, Buser D, Schenk R, Wozney J, Cochran DL. The effect of rhBMP-2 around endosseous implants with and without membranes in the canine model. J Periodontol. 2006;77(7):1184. doi:10.1902/ jop.2006.050337

25. Hing KA, Wilson LF, Buckland T. Comparative performance of three ceramic bone graft substitutes. Spine J. 2007;7(4):475-490. doi:10. 1016/j.spinee.2006.07.017

26. Kim SR, Lee JH, Kim YT, et al. Synthesis of Si, Mg substituted hydroxyapatites and their sintering behaviors. Biomaterials. 2003;24 (8):1389-1398. doi:10.1016/S0142-9612(02)00523-9

27. Shige W, Helena T, Xiangyang S. Electrospun laponite-doped poly (lactic-co-glycolic acid) nanofibers for osteogenic differentiation of human mesenchymal stem cells. J Control Release. 2012;22:23 $357-23367$.

28. Karageorgiou V, Kaplan D. Porosity of 3D biomaterial scaffolds and osteogenesis. Biomaterials. 2005;26(27):5474-5491. doi:10.1016/j. biomaterials.2005.02.002

29. Ba Z, Chen Z, Huang Y, et al. Nanoporous diopside modulates biocompatibility, degradability and osteogenesis of bioactive scaffolds of gliadin-based composites for new bone formation Int J Nanomedicine. 2018;13:3883-3896. doi:10.2147/IJN.S162262
Journal Citation Reports/Science Edition, EMBase, Scopus and the Elsevier Bibliographic databases. The manuscript management system is completely online and includes a very quick and fair peer-review system, which is all easy to use. Visit http://www.dovepress.com/ testimonials.php to read real quotes from published authors. 